\title{
Efectos de trabajar con GeoGebra en el aula en la relación afecto-cognición
}

\section{Effects of working with GeoGebra in the classroom on the affect-cognition relationship}

\author{
M. a del Mar García López, Isabel M. a Romero Albaladejo, Francisco Gil Cuadra \\ Departamento de Educación, Universidad de Almería, Almería, España. \\ mgl711@ual.es, imromero@ual.es, fgil@ual.es
}

RESUMEN • El auge del uso de la tecnología en educación matemática, especialmente en el enfoque dinámico de la geometría, requiere estudios empíricos bien fundamentados que informen sobre su uso efectivo en las aulas. Este artículo responde a la demanda de intervenciones que atiendan a las dimensiones afectiva y cognitiva, así como al estudio de la relación entre ambas. Mediante un experimento de enseńanza en dos clases de secundaria, se analiza la influencia de GeoGebra en el desarrollo de actitudes relacionadas con las matemáticas y de la competencia matemática en el alumnado. El análisis cuantitativo de los datos muestra una evolución positiva en las variables estudiadas, mientras que el análisis cualitativo informa sobre cómo se produjo esta evolución, sobre las propiedades del software que la sustentaron y sobre la relación entre los constructos afectivos y cognitivos.

PALABRAS CLAVE: Actitudes; Competencia matemática; Afecto-cognición; GeoGebra; Educación secundaria.

ABSTRACT • The rise of the use of technology in mathematics education, especially in the dynamic approach to geometry, requires well-founded empirical studies that inform its effective use in the classroom. This article responds to the demand for interventions that address the affective and cognitive dimensions, as well as the study of the relationship between them. Through a teaching experiment in two secondary school classes, the influence of GeoGebra in the development of students' attitudes related to mathematics and mathematical competence is analysed. The quantitative analysis of the data shows a positive evolution in the studied variables, while the qualitative analysis reports on how this evolution took place, on the properties of the software that supported it and on the relationship between affective and cognitive constructs.

KEYWORDS: Attitudes; Mathematical literacy; Affect-cognition; GeoGebra; Secondary education.

Recepción: abril $2020 \bullet$ Aceptación: diciembre 2020

García López, M. M., Romero Albaladejo, I. M. y Gil Cuadra, F. (2021). Efectos de trabajar con GeoGebra en elaula en la relación afecto-cognición. Enseñanza delas Ciencias, 39(3), 177-198. https://doi.org/10.5565/rev/ensciencias.3299 


\section{INTRODUCCIÓN}

Desde la aparición de la geometría dinámica, a principios de los años noventa, los desarrollos tecnológicos presentan nuevos retos en la enseñanza y el aprendizaje de la geometría. Los estudios empíricos sobre su uso en las aulas constituyen una importante línea de investigación, debido a la necesidad de comprender cómo pueden ser utilizados de forma efectiva para implicar a los estudiantes en un aprendizaje significativo (Sinclair et al., 2016).

Dentro de los sistemas de geometría dinámica (SGD), GeoGebra ha ganado terreno por ser un software libre, de rápido desarrollo y con una gran comunidad de usuarios. Además, cuenta con una trayectoria de investigación sobre su uso en las aulas, con resultados positivos tanto a nivel cognitivo como afectivo (Costa, 2011; Gómez-Chacón, Romero y García, 2016; Wassie y Zergaw, 2018; Zengìn, 2017b).

Hasta la fecha, la mayoría de los estudios sobre GeoGebra ponen el foco en el aprendizaje de distintos tópicos matemáticos y de resolución de problemas (Wassie y Zergaw, 2018). Los aspectos afectivos suelen quedar en segundo plano y, aunque se reconoce el potencial del software para fomentar la motivación de los estudiantes, su autonomía, implicación, etc., son escasos los estudios centrados en la dimensión afectiva, o los que abordan la relación afecto-cognición (Gómez-Chacón, 2011; GómezChacón et al., 2016; Gómez-Chacón y Marbán, 2019). Sin embargo, la estrecha conexión entre ambas dimensiones es manifestada por estos y otros autores (Gresalfi, 2009; Roth y Walshaw, 2019), por lo que su estudio es demandado actualmente en la agenda de investigación en educación matemática (Hannula, Leder, Morselli, Vollstedt y Zhang, 2019).

Por otro lado, Hannula et al. (2019) advierten del peligro de que los desarrollos teóricos no tengan repercusión en la práctica y reclaman programas e intervenciones que promuevan los aspectos afectivos en el aprendizaje matemático. En una etapa tan crítica como la de educación secundaria, donde la motivación y el disfrute con las matemáticas decrece con el paso de los cursos (Grootenboer y Marshman, 2015; OECD, 2010), la inclusión de herramientas tecnológicas como GeoGebra es una alternativa interesante para atender a las necesidades e intereses de los jóvenes.

Este artículo presenta un experimento de enseñanza (Molina, Castro, Molina y Castro, 2011), en el que se introduce GeoGebra en dos aulas de 3. ${ }^{\circ}$ de ESO, para trabajar contenidos de geometría plana. La finalidad de la propuesta es incidir favorablemente en las actitudes y el desarrollo competencial del alumnado, así como analizar la influencia del trabajo con el software en ambos aspectos y la relación entre ellos.

\section{MARCO TEÓRICO}

Presentamos en este apartado los constructos teóricos utilizados para abordar las dimensiones actitudinal y competencial en este estudio, que permitirán delimitar su propósito y las preguntas de investigación.

\section{La dimensión actitudinal}

El estudio del afecto en matemáticas es un campo complejo, debido a la ausencia de un marco teórico unificado y con elementos bien delimitados (Hannula, Pantziara y Di Martino, 2018). Dentro de este campo, el concepto de actitud es uno de los más problemáticos, ya que los estudios existentes sobre actitudes no concluyen una definición clara del constructo en sí mismo. Con frecuencia, la actitud es definida implícitamente, y a posteriori se nos presenta a través de instrumentos usados para medirla. Además, las investigaciones que aportan una definición de actitud no comparten una única caracterización (Di Martino y Zan, 2010). 
A pesar de esta falta de uniformidad, se observa una división clara en la literatura entre las actitudes hacia la matemática, de carácter más marcadamente afectivo y cuyo objeto es la matemática como disciplina, y las actitudes matemáticas, de carácter más cognitivo y que tienen por objeto los procesos y actividades matemáticas (De Bellis y Goldin, 2006; Gómez-Chacón, 2011).

Dentro de las actitudes hacia la matemática se incluyen la motivación y el disfrute, la autoconfianza, la utilidad percibida, el interés, la satisfacción, etc. Su estudio tiene una larga tradición, que es más reducida para los entornos tecnológicos. En el caso de GeoGebra, trabajos como los de Wassie y Zergaw (2018), Yoganci (2018), Zetriuslita, Nofriyandi e Istikomah (2020) y García y Romero (2020) muestran que este software ayuda a aumentar la confianza, la autoestima, el interés y la motivación de los estudiantes. De acuerdo con Gómez-Chacón (2011), la tendencia para evaluar las actitudes hacia las matemáticas ha sido el uso de cuestionarios, desarrollados desde la perspectiva de una definición multidimensional de la actitud, siendo la motivación y la confianza las dimensiones con mayor impacto en el aprendizaje. La propia autora señala la coincidencia de varios estudios en que la confianza y la motivación de los estudiantes tienen una correlación más fuerte con las actitudes hacia el uso de ordenadores en el proceso de enseñanza-aprendizaje que con las actitudes hacia la matemática. Abordamos ambas actitudes en este estudio.

Por lo que respecta a las actitudes matemáticas, su investigación está bastante menos desarrollada que la de las actitudes hacia la matemática (Gómez-Chacón, 2019). Ejemplos de actitudes matemáticas son la perseverancia, la flexibilidad de pensamiento, la sistematicidad, el espíritu crítico, la precisión, el rigor, etc. (De Bellis y Goldin, 2006; Gómez-Chacón, 2011, 2019). El que un estudiante muestre actitudes favorables hacia las matemáticas no quiere decir que tenga desarrolladas las mencionadas actitudes, las cuales son consustanciales al quehacer matemático. Precisamente esta fuerte componente cognitiva de las actitudes matemáticas implica que su estudio no puede realizarse únicamente de forma declarativa, sino que ha de completarse con la observación de los comportamientos de los estudiantes mientras llevan a cabo actividades matemáticas específicas. Por otra parte, las actitudes matemáticas no son el resultado de decisiones espontáneas, sino que son hábitos mentales que pueden y deben ser inculcados en entornos de clase apropiados (Gómez-Chacón, 2011).

Algunas investigaciones revelan efectos positivos del uso de la tecnología en el desarrollo de actitudes matemáticas en los estudiantes (Jaramillo y Ruiz, 2010; Stolaki y Economides, 2018). También en el caso de GeoGebra, Gómez-Chacón (2011), García (2011) y Gómez-Chacón et al. (2016) resaltan los resultados positivos de usar tecnologías que promueven la visualización y la exploración para desarrollar la perseverancia, la autonomía, el pensamiento crítico, la precisión y el rigor, y la actitud inductiva en los estudiantes.

En ausencia de un marco definido sobre cuáles son las actitudes matemáticas básicas deseables y sus rasgos observables, proporcionamos en un trabajo precedente una caracterización operativa de varias de ellas (García, 2011). Por motivos de extensión, nos limitamos aquí a presentar los indicadores derivados de dicha caracterización para la observación de las actitudes: autonomía, creatividad, flexibilidad de pensamiento, perseverancia y precisión-rigor (tabla 1): 
Tabla 1.

Parrilla de actitudes matemáticas

\begin{tabular}{|c|c|}
\hline $\begin{array}{c}\text { Actitudes matemá- } \\
\text { ticas }\end{array}$ & Indicadores \\
\hline Autonomía & $\begin{array}{l}\text { AU1 Renuncia a pensar por sí mismo/a y prefiere recurrir a otros para que le indiquen cómo } \\
\text { proceder } \\
\text { AU2 Muestra iniciativa para superar obstáculos } \\
\text { AU3 Toma sus propias decisiones para sus propios propósitos }\end{array}$ \\
\hline Creatividad & $\begin{array}{l}\text { C1 No muestra inclinación a probar caminos o estrategias diferentes para resolver un problema } \\
\text { C2 } 2 \text { Le gusta inventar nuevas estrategias o problemas } \\
\text { C3 Propone soluciones originales y estéticas a los problemas }\end{array}$ \\
\hline $\begin{array}{l}\text { Flexibilidad de } \\
\text { pensamiento }\end{array}$ & $\begin{array}{l}\text { FP1 Cambia de opinión sobre la base de argumentos convincentes } \\
\text { FP2 Resuelve los problemas de más de una forma } \\
\text { FP3 Se interesa por la/s forma/s en que otros compañeros resuelven problemas, diferentes a la } \\
\text { suya }\end{array}$ \\
\hline Perseverancia & $\begin{array}{l}\text { P1 Ante un problema, se da por vencido fácilmente sin llegar a ninguna respuesta } \\
\text { P2 Cuando fracasa en el intento de resolver un problema, se conforma con una respuesta inco- } \\
\text { rrecta y no lo intenta otra vez. } \\
\text { P3 No abandona el problema hasta que llega a una solución que le satisface y considera correc- } \\
\text { ta }\end{array}$ \\
\hline Precisión-rigor & $\begin{array}{l}\text { PR1 No da importancia a los errores de cálculo o a las imprecisiones de la construcción geomé- } \\
\text { trica } \\
\text { PR2 Se contenta con soluciones aproximadas, sin plantearse su bondad o su adecuación } \\
\text { PR3 Pone atención a las construcciones geométricas y a los cálculos, tiene escasa tolerancia a los } \\
\text { errores e intenta averiguar si estos se deben a fallos de razonamiento }\end{array}$ \\
\hline
\end{tabular}

\section{La dimensión competencial}

A la hora de promover y evaluar el aprendizaje matemático de los estudiantes, hay una corriente internacional que se enfoca en el desarrollo de su competencia matemática (Lupiáñez y Rico, 2008; Proyecto PISA, OCDE, 2017). Esta competencia global se articula en otras siete competencias o capacidades matemáticas fundamentales: comunicación, matematización, representación, razonamiento y argumentación, diseño de estrategias para resolver problemas, uso de operaciones y lenguaje simbólico, y uso de herramientas (OCDE, 2017).

Numerosas investigaciones corroboran el potencial de la geometría dinámica para ayudar a los estudiantes en la comunicación matemática (Takaci, Stankov y Milanovic, 2015; Zengìn, 2017a); la matematización (Costa, 2011); el manejo de representaciones (Santos-Trigo, 2008; Takaci et al., 2015); la formulación de conjeturas, argumentaciones y pruebas (Baccaglini-Frank, 2019; Sinclair et al., 2016; Zengìn, 2017b), y la resolución de problemas (Sinclair y Yurita, 2008; Granberg y Olsson, 2015).

En varios de estos trabajos se explicita cómo determinadas propiedades y características de los SGD apoyan el desarrollo de estas competencias. Entre ellas, destacan las siguientes: constructividad (posibilidad de construir rápidamente ejemplos sobre los que razonar, y de actuar por ensayo-error); navegabilidad (posibilidad de explorar libre y flexiblemente); interactividad (retroalimentación inmediata que permite la toma de conciencia y conciliación de errores); interfaz (modo de capturar la acción y atención del aprendiz); precisión (ejecución de las acciones del usuario con exactitud), y arrastre (o dragging, que obliga a pensar y razonar los problemas en términos de propiedades matemáticas).

Dado que este estudio se llevó a cabo en un contexto real, dentro del marco curricular establecido (García, 2011), para evaluar el desarrollo de la dimensión competencial se adoptó el marco del Análisis Didáctico, propuesto por Rico y colaboradores para diseñar y evaluar unidades didácticas (Rico y 
Lupiáńez, 2008; Lupiáńez y Rico, 2008) en consonancia con el Proyecto PISA. Dentro de este marco, el Análisis Cognitivo permite vincular las expectativas de aprendizaje de orden superior (capacidades matemáticas fundamentales) con las capacidades específicas que se ponen en juego al realizar tareas matemáticas y asociarles un nivel de complejidad del 1 al 3 (tabla 2). Dichos niveles de complejidad se corresponden con los establecidos por Rico y Lupiáńez (2008) a partir del proyecto PISA: ${ }^{1}$

- Nivel 1 (Reproducción): Los estudiantes pueden resolver cuestiones en contextos simples o familiares donde la información relevante está claramente definida. Son capaces de hacer aplicaciones directas de conocimientos matemáticos ya estudiados. Realizan operaciones rutinarias.

- Nivel 2 (Conexiones): Los estudiantes pueden trabajar situaciones relativamente desconocidas. Son capaces de seleccionar, interpretar e integrar diferentes representaciones. Aplican estrategias simples para resolver problemas y usan secuencias de cálculos. Pueden expresar brevemente sus interpretaciones y razonamientos.

- Nivel 3 (Reflexión): Los estudiantes pueden trabajar con situaciones desconocidas. Son capaces de seleccionar y evaluar estrategias apropiadas para la resolución del problema, o diseñar otras nuevas. Poseen un alto nivel de interpretación, uniendo diferentes informaciones y representaciones, y se mueven flexiblemente entre ellas. Además, comunican sus acciones y reflexiones según sus propios hallazgos, interpretaciones y argumentaciones.

\section{PROPÓSITO DE LA INVESTIGACIÓN}

El predominio de la investigación cuantitativa sobre los efectos de la tecnología en educación matemática, tanto a nivel cognitivo como afectivo, deja un hueco en la agenda, ya que dichos efectos están ligados a cómo se produce su implementación en las aulas (Hoyles y Lagrange, 2010). De ahí la demanda de estudios, incluidos los de replicación, que identifiquen factores decisivos que determinen los beneficios en casos específicos. Dichos estudios deben estar bien diseñados metodológicamente, de acuerdo con los estándares actuales, a la vez que fundamentados en las teorías específicas de la educación matemática (Drijvers, 2018). Trabajos de este tipo, como el que presentamos, pueden avanzar respuestas a las siguientes cuestiones:

- ¿Es posible diseñar con GeoGebra un entorno de enseñanza en el que la predisposición de los estudiantes hacia el uso de la tecnología se aproveche para desarrollar favorablemente sus actitudes hacia la matemática y sus actitudes matemáticas?

- En caso afirmativo, ¿cómo influye el trabajo con GeoGebra y sus características en el desarrollo de cada tipo de actitud?

- ¿Es posible llegar a una estabilización actitudinal en los estudiantes? ¿En qué medida se mantiene cuando se deja de utilizar GeoGebra?

- ¿Cómo influye el trabajo con GeoGebra, y sus propiedades, en el desarrollo de la competencia matemática del alumnado?

- ¿Repercute el desarrollo actitudinal del alumnado en su desarrollo cognitivo? ¿De qué manera? ¿Es posible establecer una ruta de influencia positiva afecto-cognición a nivel de aula, sostenida por las propiedades y usos de GeoGebra?

1. La nomenclatura y el grado de especificidad en los niveles de complejidad se ha actualizado en los sucesivos informes PISA. No obstante, mantenemos la nomenclatura y los niveles descritos por Rico y Lupiáńez (2008), por ser más sencilla su aplicación en la evaluación de aula y ser coherente con desarrollos posteriores. 


\section{METODOLOGÍA}

Atendiendo al propósito del estudio, el paradigma de la investigación de diseño puede contribuir al cuerpo de conocimiento, y proporcionar una perspectiva detallada sobre el aprendizaje matemático de los estudiantes en su entorno natural y los mecanismos que lo influencian. Dentro de este paradigma, se realizó un experimento de enseñanza en dos clases de 3. ${ }^{\circ}$ de ESO de un centro TIC de educación pública, con 23 estudiantes cada una. La profesora encargada de la implementación es la primera autora del artículo y trabajó en colaboración con la segunda autora, investigadora universitaria. Para el experimento se diseńaron dos secuencias de tareas, la primera para ser trabajada con lápiz y papel e instrumentos de dibujo tradicionales (secuencia LP), y la segunda para ser abordada mediante GeoGebra (secuencia GG). Ambas secuencias se desarrollaron con planteamientos metodológicos comunes, siendo el empleo del software la única diferencia.

Durante 25 sesiones, distribuidas en dos meses, el alumnado trabajó en parejas sobre tareas contextualizadas que podían afrontar con conocimientos básicos de geometría y sin explicaciones previas. En la secuencia GG, cada pareja de estudiantes compartía un ordenador. De acuerdo con lo que la literatura de investigación considera como buenas prácticas en educación matemática con tecnología (Bray y Tangney, 2017), se estimuló en los estudiantes la exploración, la indagación y la colaboración; mientras que la profesora actuó como facilitadora del aprendizaje, supervisando, registrando y orquestando el aprendizaje. En total se necesitaron 13 sesiones para las tareas de LP, sobre polígonos y contenidos de geometría plana; y 12 sesiones para las tareas con GG, sobre teselaciones del plano.

Durante el proceso, se utilizaron diversos instrumentos para tomar datos tanto de forma declarativa como observacional. En el caso de las actitudes, se utilizó la escala EAHM-U (Bazán, 1997) para las actitudes hacia las matemáticas, y el cuestionario MIO (García y Romero, 2020), que fue diseñado y validado exprofeso para las actitudes hacia el aprendizaje de las matemáticas con ordenadores. Además, se recogieron opiniones del alumnado en un buzón virtual de sugerencias voluntarias, y se realizó una entrevista colectiva a cada clase. Los datos declarativos se contrastaron con observaciones en el aula a través de la parrilla de actitudes (tabla 1). Las observaciones fueron registradas por la profesorainvestigadora y validadas por un observador externo.

Para la observación del desarrollo competencial en los estudiantes, se utilizaron parrillas de observación, elaboradas específicamente para cada tarea y contrastadas por un grupo de expertos en Análisis Didáctico. A modo de ejemplo, se muestra la parrilla correspondiente a la tarea 9 de la secuencia didáctica diseñada (tabla 2):

Tarea 9: Te propongo el trabajo inverso al que has venido haciendo hasta ahora: en lugar de que busques diseños de losetas que sirvan para hacer mosaicos, te doy dos diseños de mosaicos muy famosos (figuras 1 y 2) que se encuentran en la Alhambra, para que partiendo de un cuadrado, consigas dibujarlos usando GeoGebra. (Deberás usar traslaciones, giros o simetrías).

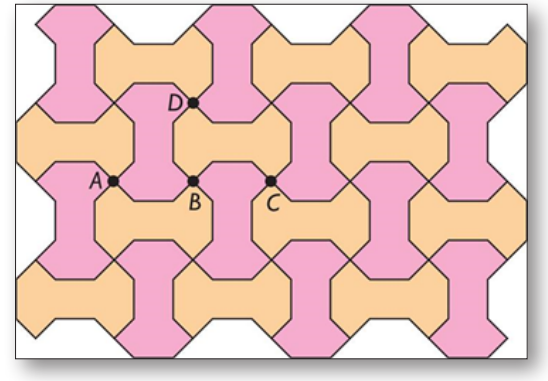

Fig. 1. Mosaico del hueso.

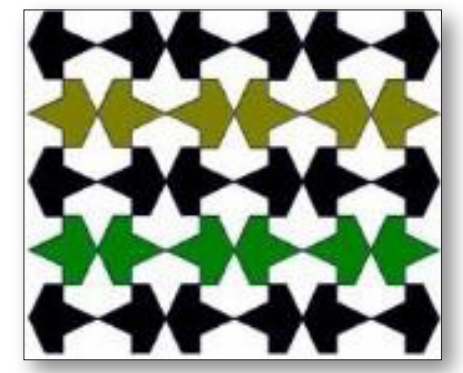

Fig. 2. Mosaico del avión. 
Tabla 2.

Parrilla de capacidades específicas, capacidades fundamentales y niveles de complejidad para la tarea 9

\begin{tabular}{|c|c|c|}
\hline & $\begin{array}{l}\text { Capacidades } \\
\text { fundamentales }\end{array}$ & Nivel \\
\hline $\begin{array}{l}\text { 1. Interpreta el enunciado del problema contextualizado en términos matemáti- } \\
\text { cos (pasar del contexto de la tarea al matemático) }\end{array}$ & $\mathrm{RA}, \mathrm{M}, \mathrm{RE}$ & 2 \\
\hline \multicolumn{3}{|l|}{ 2. Expresa oralmente y por escrito estrategias seguidas y resultados } \\
\hline 2.1 Se expresa oralmente con sus palabras & $\mathrm{C}$ & 1 \\
\hline 2.2 Se expresa oralmente con vocabulario matemático adecuado & $\mathrm{C}$ & 2 \\
\hline 2.3 Se expresa por escrito con sus palabras & $\mathrm{C}, \mathrm{RP}$ & 1 \\
\hline 2.4 Se expresa por escrito con vocabulario matemático adecuado & $\mathrm{C}, \mathrm{RP}$ & 2 \\
\hline 3. Maneja distintas representaciones de polígonos para crear mosaicos & RE, UH & 2 \\
\hline $\begin{array}{l}\text { 4. Distingue representaciones de mosaicos regulares dibujando y usando iso- } \\
\text { metrías }\end{array}$ & RA, M, RE & 2 \\
\hline $\begin{array}{l}\text { 5. Identifica y explica la obtención del motivo mínimo de un mosaico dada su } \\
\text { representación contextualizada }\end{array}$ & $\mathrm{C}, \mathrm{M}, \mathrm{RE}$ & 3 \\
\hline $\begin{array}{l}\text { 6. Crea teselas deformando por giros y/o traslaciones los lados de un triángulo, } \\
\text { cuadrilátero o hexágono regular, explicando el procedimiento seguido }\end{array}$ & C, RE, UH & 3 \\
\hline \multicolumn{3}{|l|}{$\begin{array}{l}\text { 7. Crea mosaicos a partir de polígonos deformados con distintas isometrías o } \\
\text { con combinaciones de ellas, explicando el procedimiento seguido }\end{array}$} \\
\hline 7.1 No se plantea una estrategia: busca combinaciones por ensayo-error & RA, C, RE, RP, UH & 1 \\
\hline $\begin{array}{l}\text { 7.2 Razona qué isometría debe aplicar identificando sus elementos: vector, } \\
\text { centro, ángulo, eje }\end{array}$ & RA, C, RE, RP, UH & 2 \\
\hline $\begin{array}{l}\text { 8. Argumenta cómo obtiene las teselas y los mosaicos (vectores y ángulos de } \\
\text { giro) }\end{array}$ & A, C & 3 \\
\hline $\begin{array}{l}\text { 9. Comprende razonamientos y argumentaciones de otros (compañeros o del } \\
\text { profesor) }\end{array}$ & RA, C & 3 \\
\hline $\begin{array}{l}\text { 10. Emplea correctamente las distintas herramientas isométricas del programa } \\
\text { para teselar }\end{array}$ & RE, UH & 2 \\
\hline
\end{tabular}

Nota: $\mathrm{RA}=$ Razonar, $\mathrm{A}=$ Argumentar, $\mathrm{C}=$ Comunicar, $\mathrm{M}=$ Matematizar, $\mathrm{RE}=$ Representar, $\mathrm{RP}=$ Diseño de estrategias para resolución de problemas, $\mathrm{UH}=$ Uso de herramientas.

La secuencia completa de tareas GG, con sus correspondientes parrillas, pueden consultarse en García (2011, pp. 654-666).

Los datos procedentes de los instrumentos anteriores se analizaron para la totalidad de estudiantes. Además, se hizo un estudio en profundidad de una muestra de 12 de ellos, representativa del total, seleccionada a partir de los perfiles actitudinales y cognitivos identificados durante la secuencia de LP (tabla 3). 
Tabla 3.

Perfiles de la muestra de estudiantes

\begin{tabular}{|c|l|c|c|c|}
\hline \multirow{2}{*}{} & \multirow{2}{*}{} & \multicolumn{2}{|c|}{ Actitudes } \\
\cline { 3 - 5 } & & Inadecuadas & Adecuadas & Buenas \\
\hline \multirow{3}{*}{ Competencia } & Insuficiente & $\mathrm{A} 2, \mathrm{~A} 3, \mathrm{~A} 7, \mathrm{~A} 9$ & $\mathrm{~A} 5, \mathrm{~A} 11$ & \\
\cline { 2 - 5 } & Suficiente & $\mathrm{A} 6$ & $\mathrm{~A} 1, \mathrm{~A} 8, \mathrm{~A} 12$ & $\mathrm{~A} 10$ \\
\cline { 2 - 5 } & Buena & & & $\mathrm{A} 4$ \\
\hline
\end{tabular}

Para estos estudiantes, se triangularon los datos procedentes de las soluciones escritas a las tareas, los archivos de su resolución con GeoGebra, las grabaciones en audio del proceso de resolución y las parrillas de observación anteriormente mencionadas, mediante el software Atlas.ti (figura 3).

En dicho software se insertaron las grabaciones de audio, los archivos de GeoGebra y los protocolos de resolución de cada tarea para cada estudiante de la muestra, integrados de forma que su trabajo durante cada sesión pudiera ser reconstruido. A continuación, se codificó el material, tomando como códigos los indicadores de las parrillas de actitudes y competencias. Se añadieron los datos declarativos relativos a las actitudes hacia la matemática y los comentarios realizados por la profesora-investigadora en su diario de clase. La codificación y el análisis de cada episodio significativo permitieron comprender el proceso evolutivo de actitudes y competencias, y establecer conexiones. Asimismo, permitieron observar qué factores apoyaban este proceso (propiedades de GeoGebra, interacción con compañeros e interacción con profesora). Este trabajo fue revisado y aprobado por un experto en Atlas.ti y en análisis del discurso en el aula. 


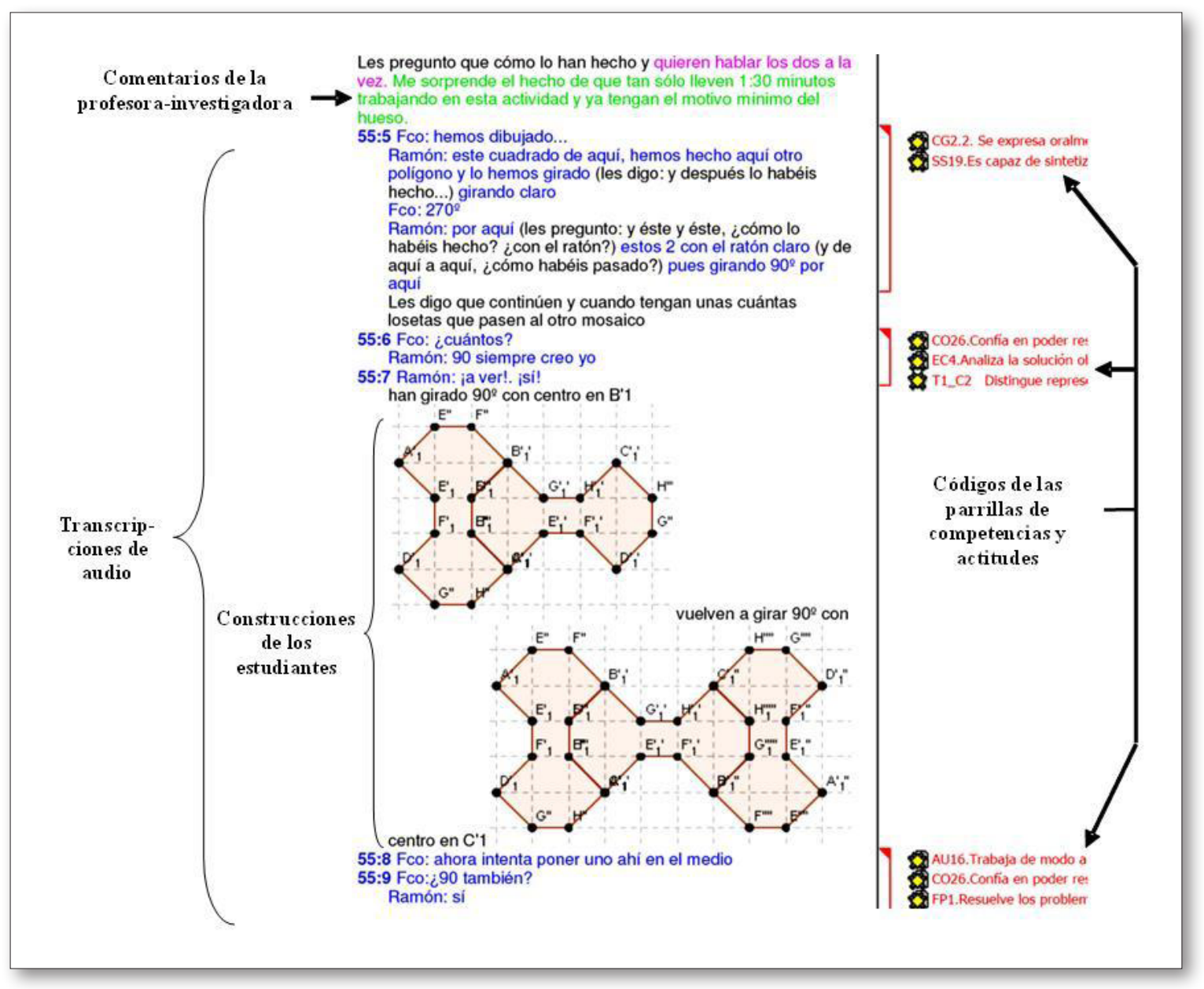

Fig. 3. Codificación y análisis de un fragmento del trabajo de una pareja de estudiantes con Atlas.ti.

\section{RESULTADOS Y DISCUSIÓN}

La triangulación de datos cuantitativos y cualitativos permitió la construcción de una imagen consistente y detallada de los efectos del uso de GeoGebra en el aula, que presentamos y discutimos a continuación.

\section{Desarrollo actitudinal con GeoGebra}

Por lo que respecta a las actitudes hacia la matemática, los análisis estadísticos de las respuestas a la escala EAHM-U, completada antes y después de la experiencia con GeoGebra, revelaron que la mayoría de los estudiantes no experimentó mejoras en sus actitudes hacia las matemáticas. Sin embargo, cuando se les preguntó específicamente por estas actitudes durante el trabajo con GeoGebra mediante el cuestionario MIO, el $63 \%$ de los estudiantes indicaron que el uso de los ordenadores les había ayudado a sentirse más seguros; el 72,1 \% afirmó haber confiado más en sus capacidades y el 78,3 \% señaló que el uso de GeoGebra les había motivado para trabajar en matemáticas (García y Romero, 2020). Estos resultados concuerdan con los anteriormente expuestos sobre la correlación más fuerte de la confianza y la motivación con las actitudes hacia la tecnología que con las actitudes hacia las matemáticas. 
La triangulación de la información procedente de los restantes instrumentos empleados corrobora el resultado de los test. Así, en los buzones virtuales de sugerencias con las opiniones libres de los estudiantes, el 90,5 \% de ellos expresó que el manejo de la herramienta ayudó a que les gustase más el trabajo en matemáticas, les resultase más fácil y mostrasen más confianza en sus posibilidades de éxito, como se aprecia en estas entradas del buzón:

- Opinión de J. P.: «Me ha gustado mucho lo de hacer las mates con el ordenador porque así mejoramos nuestro rendimiento, nos gustan más, se nos pasa más rápido el tiempo y nos esforzamos más y nos lo pasamos mejor».

- Opinión de M. R.: «Me ha gustado trabajar con los ordenadores, me ha parecido muy interesante y se me ha dado mejor. La asignatura se me ha hecho más amena y me ha resultado mucho más fácil. Sí, me ha gustado mucho».

También en las entrevistas grupales los estudiantes informaron sobre la motivación y la confianza promovidas por el SGD:

- Pregunta: ¿Qué es lo más importante o significativo de la experiencia de estudiar geometría con GeoGebra?

- Respuesta de E. S.: "Con los ordenadores estás más motivado. Cuando lo hacíamos con LP, antes de la clase pensabas que tocaba matemáticas y estabas desganado, pero con los ordenadores nos gusta más. (Varios compańeros opinan igual)»

- Respuesta de R. B.: «Es más entretenido. Con el ordenador se ve mejor porque puedes hacerlo tú, sacar la conclusión tú».

El análisis más completo llevado a cabo para los estudiantes de la muestra corrobora estos resultados. Así, el análisis de sus parrillas de actitudes informó de una mayor motivación (mayor gusto por las matemáticas) y de un aumento de la confianza en sus posibilidades de éxito propiciada por el trabajo con GeoGebra (figuras 4 y 5, respectivamente):

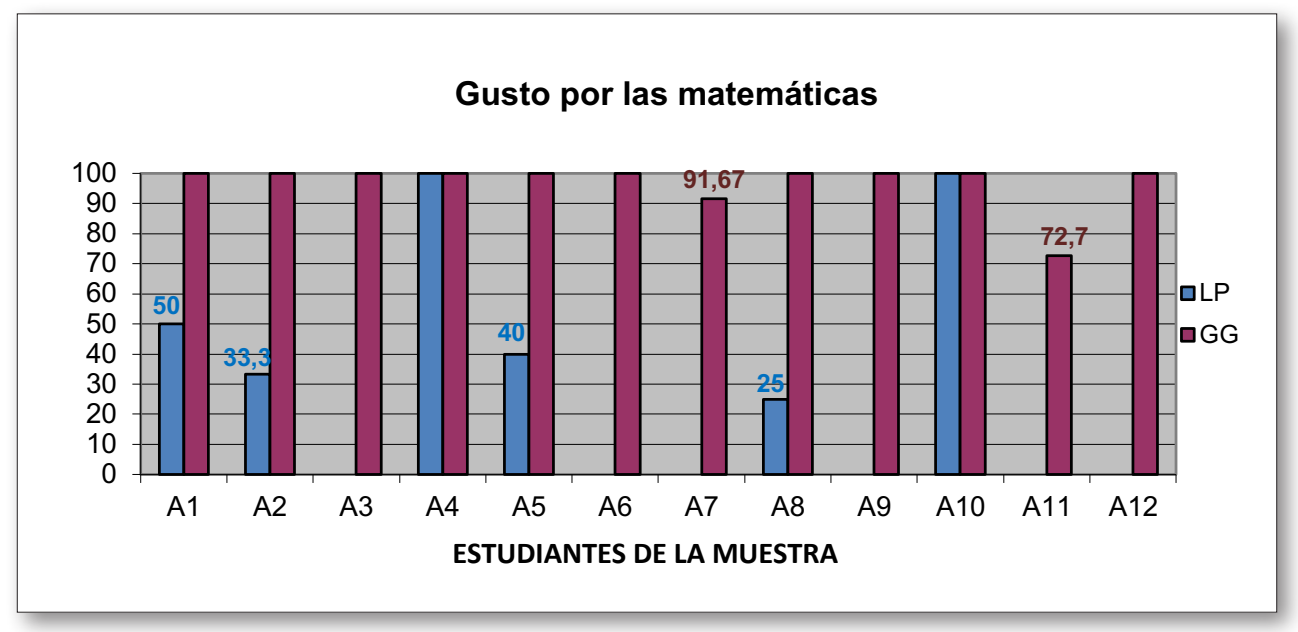

Fig. 4. Porcentajes medios obtenidos por los estudiantes de la muestra durante las tareas LP y GG en el gusto por las matemáticas. 


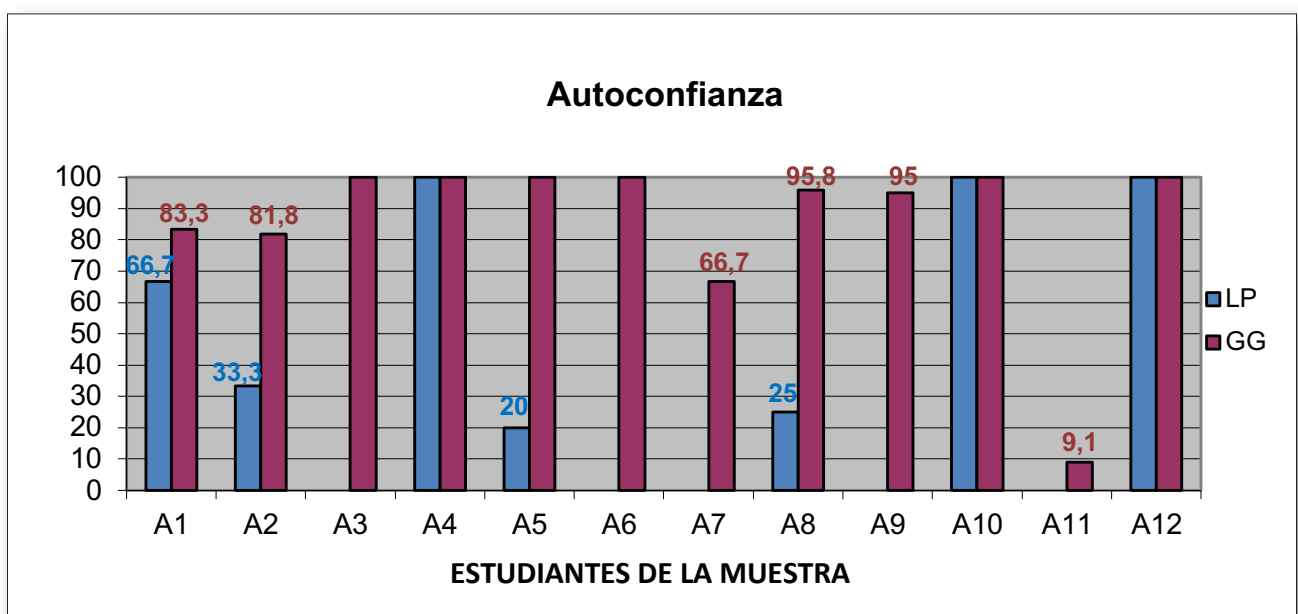

Fig. 5. Porcentajes medios obtenidos por los estudiantes de la muestra durante las tareas LP y GG en autoconfianza.

En el caso de las actitudes matemáticas, los resultados cuantitativos para el total de estudiantes (tabla 4) muestran un claro progreso en las sesiones GG. Durante las tareas LP, los alumnos mostraban escasas actitudes matemáticas, siendo las más desfavorecidas autonomía y creatividad. Con la introducción del software, tres de las cinco actitudes mejoraron notablemente: perseverancia, autonomía y precisiónrigor. En menor medida evolucionaron la flexibilidad de pensamiento y la creatividad.

Tabla 4.

Actitudes matemáticas en sesiones LP y GG

\begin{tabular}{|l|c|c|c|c|c|c|}
\hline \multirow{2}{*}{ Actitud } & \multicolumn{3}{c|}{ Lápizypapel $^{a}$} & \multicolumn{3}{c|}{ GeoGebra $^{a}$} \\
\hline & Ninguno & Algunos & Mayoría & Ninguno & Algunos & Mayoría \\
\hline Autonomía & 72 & 28 & 0 & 4 & 0 & 96 \\
\hline Creatividad & 68 & 27 & 5 & 25 & 42 & 38 \\
\hline Flexibilidad de pensamiento & 51 & 34 & 15 & 21 & 12 & 67 \\
\hline Perseverancia & 55 & 40 & 5 & 0 & 4 & 96 \\
\hline Precisión y rigor & 50 & 45 & 6 & 8 & 0 & 92 \\
\hline
\end{tabular}

a) porcentaje de sesiones con lápiz y papel y con GeoGebra en las cuales ningún estudiante, algunos estudiantes (menos del $70 \%$ del total) o la mayoría de ellos (70 \% o más del total) manifestó cada una de las actitudes estudiadas.

Por lo que respecta a los 12 estudiantes de la muestra, la figura 6 muestra de forma simplificada los resultados obtenidos a partir de las parrillas de observación individuales. Los porcentajes se obtuvieron analizando el porcentaje de sesiones en las que los estudiantes de la muestra manifestaron adecuadamente cada actitud, tanto para las sesiones de LP como para las de GG. 


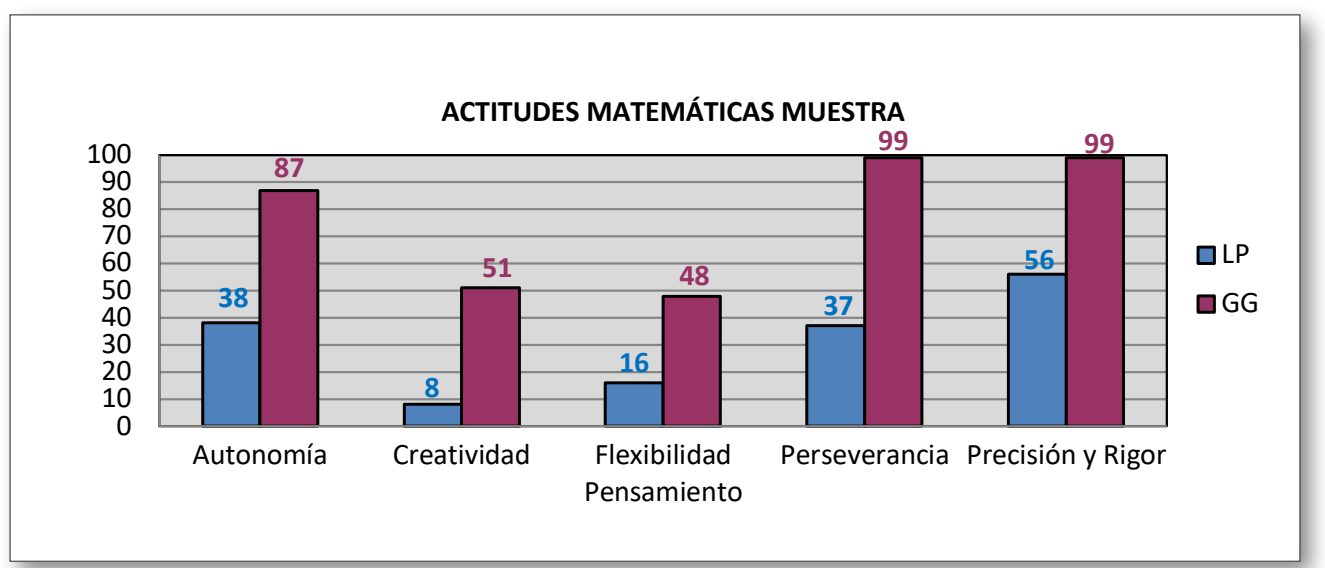

Fig. 6. Porcentajes de sesiones y actitudes evidenciadas por estudiantes de la muestra.

\section{Factores de influencia en el desarrollo actitudinal}

Respecto a los atributos de GeoGebra que influyeron en el desarrollo actitudinal, la constructividad ejerció mayor influencia para el desarrollo de la confianza de los estudiantes. Así, poder probar construcciones y tener actividad en todo momento los incitó a continuar trabajando en las tareas, y el ir comprobando cómo eran capaces de resolverlas les fue haciendo sentirse más seguros. También la interactividad del SGD contribuyó a ello, ya que la realimentación en tiempo real cuando probaban alguna idea, incluso en ausencia de una estrategia definida, ayudó a muchos estudiantes a vencer las situaciones de bloqueo, al tiempo que les informaba de la bondad de algunas de sus acciones, haciéndoles ganar poco a poco confianza en poder resolver las tareas por sí mismos. El siguiente fragmento extraído de la reconstrucción con Atlas.ti de la tarea 1 para A7 puede tomarse como ejemplo:

A7: «yo quiero hacer aquí una figura, pero no sé... voy a probar figuras ahí a mogollón».

A7: «Espérate (se dice a sí misma), vamos a concentrarnos».

A7: «¿Y qué era un pentágono? (se ríe y sigue hablando consigo misma y se para a pensar cómo continuar. Se muestra muy animada para encontrar más mosaicos y parece que la tarea no le disgusta)».

A7: «Yo no sé qué estoy haciendo aquí, pero conseguiré hacer una figura. ¡Vamos que si la hago!» (se muestra bastante confiada en que logrará su objetivo).

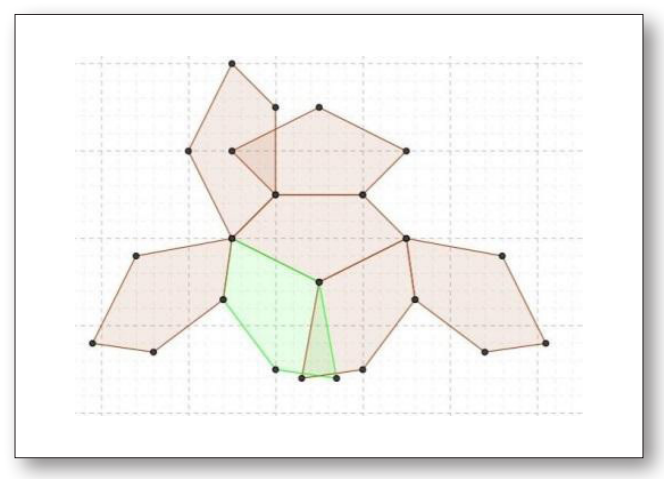

Fig. 7. Ejemplo del apoyo de GeoGebra al desarrollo de la autoconfianza en A7. 
En el caso de las actitudes matemáticas, GeoGebra fue el factor más influyente en las de precisiónrigor, perseverancia y autonomía, que todos los estudiantes manifestaron de forma continuada. También fomentó la flexibilidad de pensamiento y, en menor medida, la creatividad durante todas las sesiones para algunos estudiantes. Sin embargo, para estas dos últimas actitudes no puede atribuirse la mejora solo al uso del recurso, sino que cobraron relevancia los factores sociales; a saber, la interacción con la pareja y con la profesora.

Por lo que respecta a las propiedades de GeoGebra que apoyaron las actitudes matemáticas, se observó que la constructividad procuró a los estudiantes una mayor perseverancia y autonomía. Durante la secuencia LP, la mayor parte de ellos se bloqueaba después de leer el enunciado de la tarea, y solicitaba ayuda inmediatamente. Con GeoGebra, el poder actuar por ensayo-error, en ausencia de una estrategia de resolución definida, así como el poder generar gran cantidad de ejemplos sobre los que razonar y argumentar de un modo sencillo, los mantuvo trabajando hasta concluir las tareas. También la navegabilidad favoreció que los estudiantes se responsabilizaran del propio trabajo, explorando de forma libre y flexible distintas ideas. Por otra parte, la interactividad (retroalimentación inmediata y efectiva proporcionada en tiempo real) adecuó el ritmo de trabajo a la situación particular de cada pareja, lo que favoreció la colaboración entre ellos y la autonomía respecto de la profesora.

El siguiente fragmento del diario de la profesora-investigadora muestra la perseverancia en dos estudiantes de la muestra:

Me sorprendió una conversación entre A7 y A8 durante la tarea 10. Ambos estaban frustrados por no haber acabado la tarea, pese a llevar toda la sesión intentándolo. Después de muchos intentos fallidos, no abandonaron la tarea, sino que demostraron mayor perseverancia, al plantearse instalar GeoGebra en sus casas y seguir intentándolo. A8 así lo hizo y me envió la tarea resuelta por e-mail (figura 8). A7, aunque lo intentó, tampoco consiguió resolverla en casa y, al no sentirse satisfecha con su trabajo, no la envió.

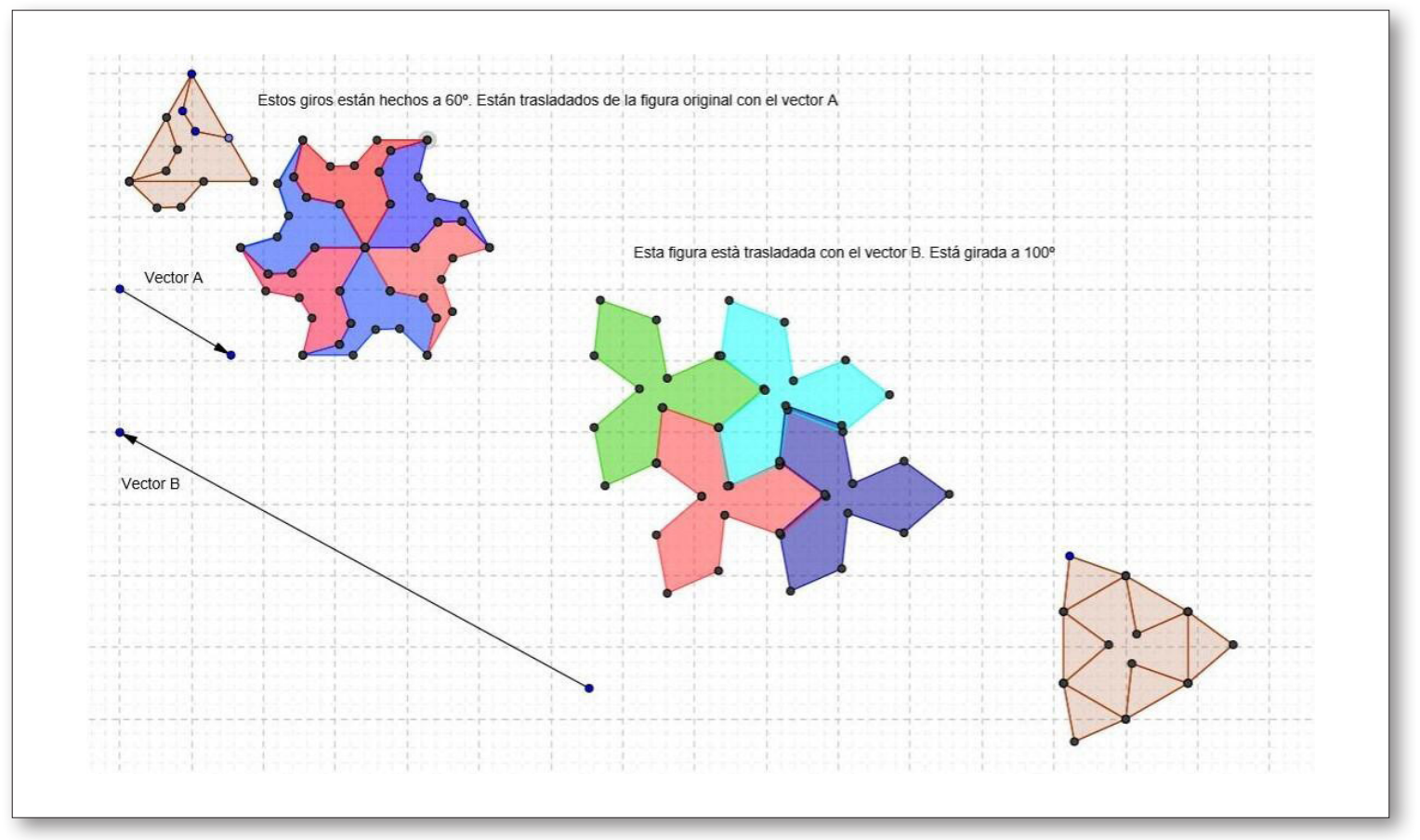

Fig. 8. Ejemplo del apoyo de GeoGebra en el desarrollo de la perseverancia de A8. 
También la interactividad de GeoGebra, junto al interfaz y la precisión al ejecutar los cálculos, contribuyeron a que los estudiantes mostraran una actitud de rigor. La visualización precisa de los efectos de sus acciones los ayudó a tomar conciencia de los errores, y la rapidez de respuesta redujo el esfuerzo y tiempo necesarios para enmendarlos. A diferencia de lo que ocurría en las tareas LP, donde muchos estudiantes se contentaban con respuestas aproximadas, con el programa pudieron rehacer y deshacer las tareas tantas veces como fue necesario hasta lograr la precisión deseada. Con el tiempo, ello les incitó a plantearse si las estrategias empleadas eran adecuadas o no, lo que fomentó un comportamiento mucho más riguroso que el exhibido anteriormente. Estos mismos factores animaron a los estudiantes a buscar distintas vías de solución de las tareas y a cambiar de proceder convenientemente, y de forma razonada, contribuyendo así a desarrollar la flexibilidad de pensamiento. Al mismo tiempo, aquellos más creativos encontraron la manera de explotar estas propiedades para realizar producciones originales.

\section{Permanencia de las transformaciones actitudinales}

Las observaciones sistemáticas realizadas por la profesora-investigadora previamente, durante y después del trabajo con GeoGebra permiten informar sobre la permanencia de las transformaciones actitudinales experimentadas por los estudiantes. En este sentido, hay diferencias respecto a los dos tipos de actitudes consideradas. Así, la transformación favorable de las actitudes hacia la matemática estuvo ligada al uso de GeoGebra y no se extrapoló a la materia al acabar el experimento. Aquellos estudiantes con mala actitud inicial (en torno al $35 \%$ del total), que el uso del software ayudó a revertir, a veces de un modo sorprendente, volvieron a manifestar actitudes hacia las matemáticas inadecuadas, algunos de forma inmediata y otros de forma más progresiva.

En cuanto a las actitudes matemáticas, es interesante notar que la herramienta no solo ayudó a su mejora, sino también a que los estudiantes tomasen conciencia de su importancia para la actividad matemática. En unidades didácticas posteriores, intentaron mantener estas actitudes sin la ayuda del software (especialmente la perseverancia y la precisión-rigor), tal como refleja la siguiente entrada en el diario de clase de la profesora-investigadora:

Al finalizar la unidad didáctica trabajada con GeoGebra, y continuar la siguiente con lápiz y papel (cuerpos geométricos), observo cómo muchos de los estudiantes que mejoraron actitudinalmente con GeoGebra siguen comportándose de este modo. Se siguen mostrando perseverantes y no abandonan las tareas hasta obtener una respuesta que les satisfaga. Además, tratan de hacer los cálculos y representaciones de la manera más rigurosa y precisa posible, si bien las representaciones gráficas de cuerpos geométricos y sus desarrollos planos sin el software les resultan algo complejas a muchos de ellos, y piden ayuda externa (lo que merma su autonomía).

No a todos los estudiantes les resultó sencillo seguir manteniendo estas actitudes sin el soporte de GeoGebra, y sin el plus de motivación y confianza que les aportaba. Una parte de ellos fueron abandonándolas conforme avanzó el curso, aunque de modo más lento y progresivo que en el caso de las actitudes hacia las matemáticas.

\section{Desarrollo de la competencia matemática con GeoGebra y factores de influencia}

El análisis del desarrollo de la competencia matemática se realizó para todo el alumnado a partir de la información recogida en las parrillas de competencias (tabla 2), los protocolos escritos de resolución entregados por cada pareja de estudiantes, los archivos de GeoGebra y las observaciones recogidas por la profesora-investigadora en sus diarios. En la tabla 5 se muestra el nivel de desarrollo de la compe- 
tencia matemática de los estudiantes al término de la experiencia, de acuerdo con los niveles anteriormente expuestos:

Tabla 5.

Nivel de desarrollo de la competencia matemática en los estudiantes

\begin{tabular}{|c|c|c|}
\hline & \multicolumn{2}{|c|}{ Competencia matemática } \\
\hline Nivel & LP & GG \\
\hline 0 & 28 & 2 \\
\hline 1 & 35 & 18 \\
\hline 2 & 28 & 50 \\
\hline 3 & 9 & 30 \\
\hline
\end{tabular}

Nota. Valores expresados en porcentajes

En torno al $80 \%$ de los estudiantes (37 estudiantes de 46) alcanzaron un nivel medio o superior, es decir, un nivel de conexión o reflexión. Así, la mitad del alumnado consiguió matematizar y resolver los problemas mediante la utilización de procedimientos y aplicaciones estándar, pero también a través de procedimientos que les llevaron a establecer conexiones entre distintas formas de representación y comunicación (tablas, gráficos, palabras e ilustraciones). Además, 14 de esos 37 estudiantes alcanzaron un nivel superior (aproximadamente el $30 \%$ del total) que les permitió reflexionar sobre sus estrategias, razonamientos y resultados. A partir de varios ejemplos, consiguieron extraer reglas, hacer generalizaciones y completar argumentos deductivos.

En cuanto a las capacidades matemáticas fundamentales, en un trabajo precedente informamos del desarrollo específico de las siguientes: uso de herramientas, representaciones, matematización, resolución de problemas, razonamiento y argumentación (Romero, García y Codina, 2015). Resumidamente, informamos de que GeoGebra resultó ser muy intuitivo y fácil de usar. El alumnado alcanzó niveles adecuados de destreza en el manejo de la herramienta desde el principio, prácticamente sin instrucción previa. Coincidimos con Costa (2011) y Gómez-Chacón (2010) en que el mayor rendimiento se obtuvo en las destrezas más visuales y manipulativas.

A continuación, se desarrollaron las capacidades de matematización y resolución de problemas, en las que el alumnado alcanzó un buen nivel (2-3), mediante el uso del software. Así, la posibilidad de manipular representaciones les permitió comprobar, a menudo por ensayo y error, qué propiedades se mantenían invariantes para cada construcción. Ello, junto con el tiempo extra proporcionado por la rapidez de la herramienta, fomentó la capacidad de razonar en términos de propiedades geométricas. Por otra parte, gracias a la interactividad del SGD, los estudiantes pudieron comprobar de forma inmediata lo apropiado, o no, de sus acciones. Con el tiempo, un buen número de ellos abandonó su anterior costumbre de realizar acciones al azar. Progresivamente, sus procesos mentales se redirigieron a planificar metas e idear estrategias para alcanzarlas.

A partir de aquí, varios estudiantes consiguieron hacer razonamientos complejos y concebir argumentos y pruebas de calidad creciente. No obstante, en las capacidades ligadas a los procesos reflexivos y discursivos el desarrollo fue más lento, y no todos los estudiantes alcanzaron niveles apropiados. Conforme aumentó la demanda de razonamiento lógico, argumentación y empleo de lenguaje matemático preciso, se evidenció la importancia de los factores sociales: la interacción con los compañeros, la interacción con la profesora y la manera en la que esta orquestó las dinámicas que surgían en clase a partir de las tareas propuestas. 
El siguiente extracto ilustra el desarrollo a nivel 2 de las capacidades 2 y 7 (tabla 2), asociadas al diseño de estrategias para resolver problemas en el alumno A3 de la muestra, que partía de una situación previa muy negativa. No había manifestado ninguna actitud matemática adecuada antes del trabajo con GeoGebra y su rendimiento en la asignatura era deficiente.

Para resolver la tarea 9, primero, obtiene el mosaico del hueso con giros y traslaciones. Después, para el mosaico del avión, prueba a construirlo con el ratón, tratando de dibujar la pieza sobre un cuadrado, marcando los vértices sobre la grilla. Luego cambia de estrategia y dibuja dos triángulos y los gira, pero al apreciar que no le sale perfecto, los borra. Comienza de nuevo hallando el centro del cuadrado, después dibuja un triángulo situando uno de sus vértices sobre dicho punto central y los otros dos vértices sobre uno de los lados del cuadrado. Finalmente rota este triángulo 4 veces hasta obtener el motivo mínimo del avión. Por último, mediante giros de $180^{\circ}$ y traslaciones, obtiene el mosaico completo. No sólo obtiene ambos mosaicos usando distintas estrategias, sino que lo argumenta en términos matemáticos correctos, oralmente y por escrito. Continúa trabajando de modo riguroso, mostrando gran autonomía, sistematización y perseverancia. Además, disfruta trabajando así.

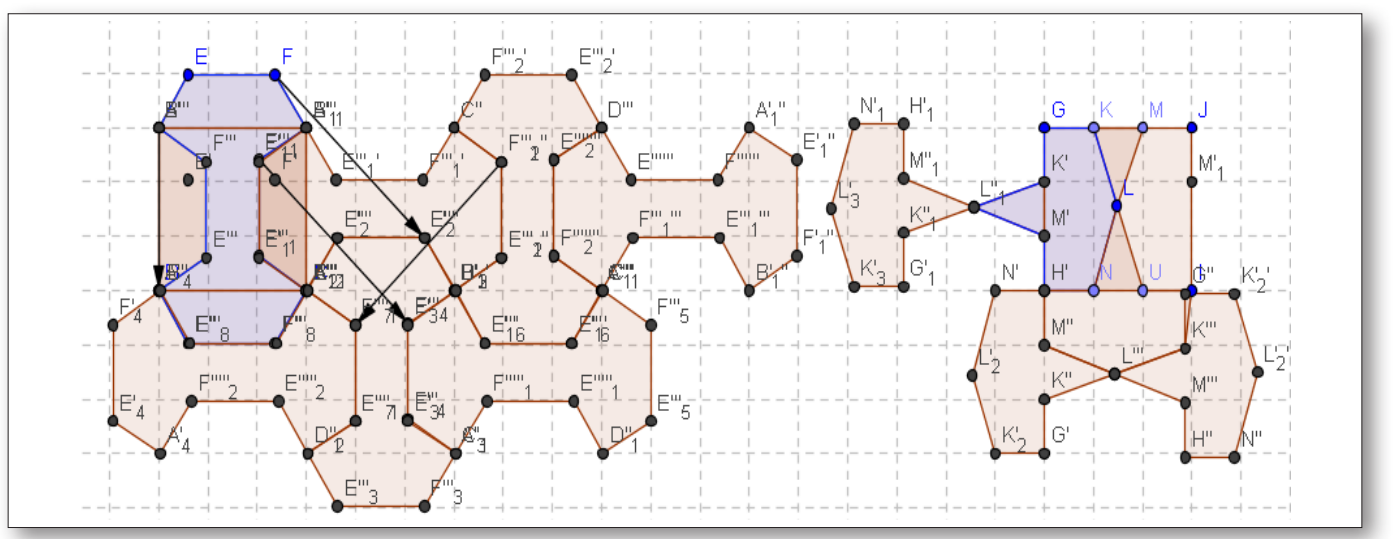

Fig. 9. Ejemplo del desarrollo de la capacidad fundamental Diseñar estrategias para resolver problemas de A3.

\section{Ruta de interacción afecto-cognición}

El análisis de datos a nivel de clase muestra un aumento de la motivación del alumnado hacia las matemáticas vinculado al uso de GeoGebra. Este aumento de la motivación y el gusto por la actividad matemática vinieron acompañados del aumento de la confianza en la propia capacidad de resolver problemas mediante la herramienta. El análisis global también informa de una evolución notable de la perseverancia, autonomía y precisión-rigor en el alumnado y, en menor medida, de la flexibilidad de pensamiento y la creatividad. Asimismo, se constata la mejora de la competencia matemática, siendo muy patente en las capacidades de manejo del recurso y de las representaciones, notable en las de matematización y resolución de problemas, y menos evidente en las de razonamiento y argumentación.

El análisis de datos con Atlas.ti para los estudiantes de la muestra, además de informar de los factores de influencia en las mejoras anteriormente indicadas, mostró una relación de dependencia entre la evolución actitudinal y la competencia matemática. Desde el principio, la motivación hacia el trabajo con GeoGebra y la confianza en su uso impulsaron a la mayoría de los estudiantes a implicarse más en las tareas, a disfrutar de ellas y a confiar en sus posibilidades de resolverlas, perseverando en sus intentos. Al mismo tiempo, despertó en ellos la necesidad de realizarlas de modo riguroso y preciso. De este modo, la motivación y la confianza al trabajar la geometría con GeoGebra, junto con las pro- 
piedades del software, resultaron fundamentales como activadoras del ámbito cognitivo, al propiciar que la mayoría de los estudiantes (más de un $70 \%$ ) mantuvieran niveles muy altos de perseverancia, autonomía y precisión-rigor, que contrastaban con los manifestados previamente. Para las actitudes matemáticas de flexibilidad de pensamiento y creatividad, las propiedades del software y las interacciones sociales ejercieron un mayor peso en su transformación positiva que el hecho de disfrutar y mostrarse motivados y confiados en matemáticas. Por otra parte, las actitudes hacia la matemática, mediadas por GeoGebra, tuvieron un desarrollo más rápido y menos estable al finalizar el uso de la herramienta que las actitudes matemáticas. El desarrollo actitudinal descrito constituyó una base sobre la que se fue desarrollando la competencia matemática del alumnado, de forma progresiva y fruto de la transformación actitudinal previa.

Por otra parte, la facilidad de uso de GeoGebra proporcionó a los estudiantes soporte suficiente para alcanzar un buen nivel de desempeño en las capacidades relacionadas con la visualización, la construcción y el manejo de representaciones. Ello les permitió involucrarse en procesos más complejos de comunicación matemática, matematización y resolución de problemas con sus compañeros. La autonomía de las parejas de estudiantes posibilitó a la profesora-investigadora ayudarlos de modo particular en las dificultades surgidas, especialmente en los procesos superiores vinculados a la capacidad de razonamiento y argumentación (Gómez-Chacón et al., 2016). La figura 10 esquematiza esta ruta de interacción afecto-cognición representativa del conjunto de la población estudiada, si bien estudiantes concretos presentaron variaciones particulares.

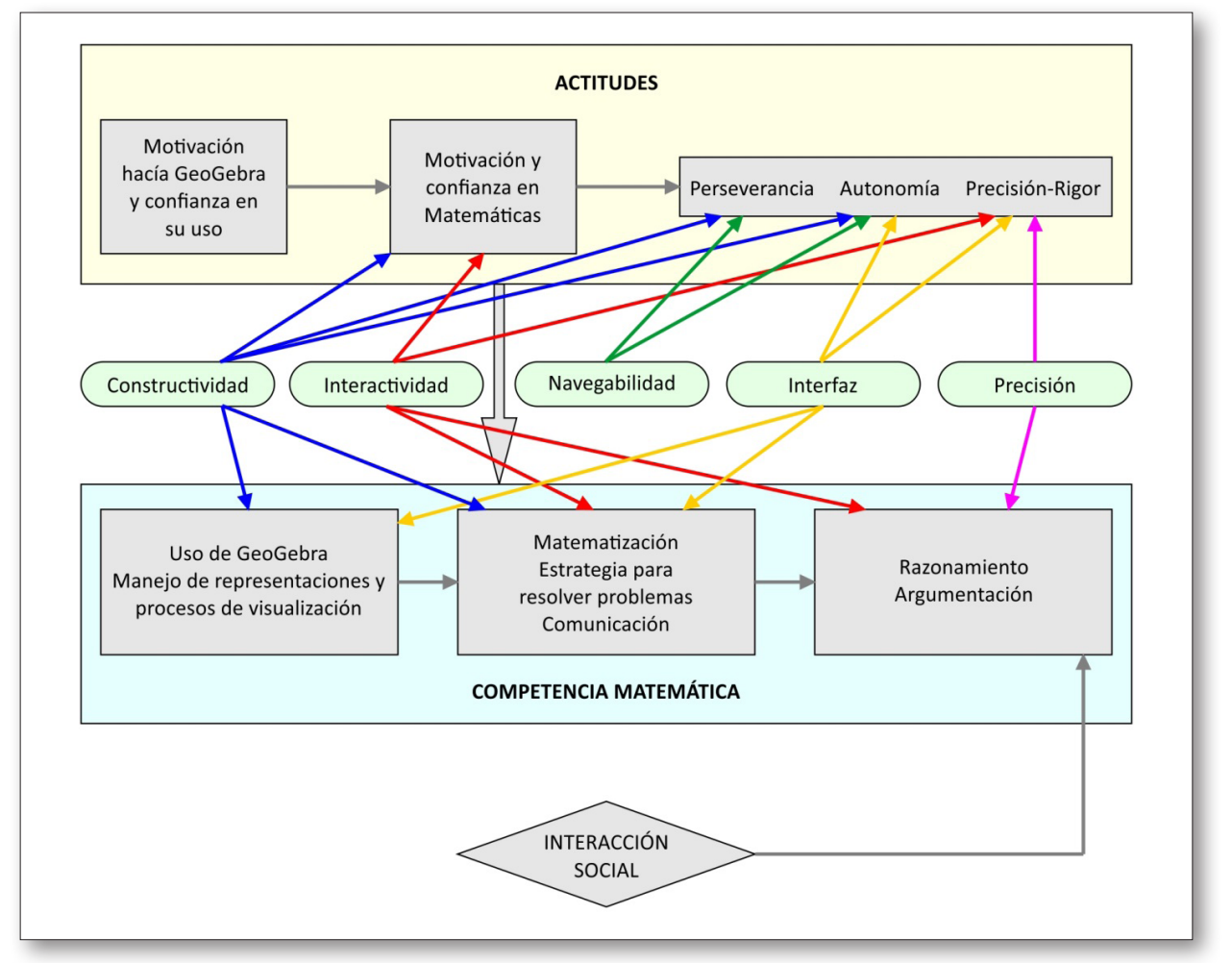

Fig. 10. Ruta de interacción afecto-cognición.

Las implicaciones señaladas resultaron más significativas para los estudiantes con actitudes hacia las matemáticas y competencia matemática previas negativas, que gracias al uso de GeoGebra lograron revertir. Al analizar a aquellos que mejoraron únicamente durante la secuencia con GeoGebra (16 
estudiantes), se comprobó que fueron precisamente los que, debido al gusto y confianza depositado en la herramienta, experimentaron un sorprendente cambio en sus actitudes hacia las matemáticas, lo que provocó una mejora de sus actitudes matemáticas y de su competencia. Al dejar de trabajar con GeoGebra volvieron a manifestar actitudes hacia las matemáticas inadecuadas que los llevaron a no trabajar lo suficiente, con lo que sus actitudes y competencias matemáticas empeoraron y, por ende, también su calificación en unidades didácticas posteriores.

\section{CONCLUSIÓN}

En un mundo que nos aboca a integrar la tecnología en la educación a pasos agigantados, con programas de software libre como GeoGebra fácilmente accesibles, el experimento de enseñanza presentado contribuye a avanzar algunos puntos pendientes en la agenda. Por un lado, responde a la necesidad de compartir intervenciones en el aula que informen de la potencialidad de los SGD en la práctica. Esto minimiza el riesgo de que la investigación sobre innovaciones tecnológicas permanezca en la periferia del uso en las clases, perdiendo así su potencial transformativo (Wassie y Zergaw, 2018).

Por otro lado, este trabajo atiende a una doble demanda en la investigación sobre afecto en educación matemática: a) informar sobre el vínculo entre constructos afectivos y su conexión con la cognición, en estudios donde la actividad matemática tenga relevancia explícita en los aspectos afectivos investigados, y $b$ ) ligar la teoría con la práctica de modo que la primera sea útil para transformar la segunda (Hannula et at., 2019).

Atendiendo a los interrogantes planteados para esta investigación, se ha diseñado una secuencia de tareas basada en el uso GeoGebra, junto con sus instrumentos de evaluación de actitudes y competencia matemática, fundamentados en la teoría y avalados por expertos. El análisis de los datos obtenidos a partir de estos instrumentos nos informa de que es posible capitalizar las actitudes favorables del alumnado hacia las matemáticas con GeoGebra, en especial motivación y autoconfianza, para desarrollar actitudes matemáticas, inherentes a la competencia matemática. Con el tipo de tareas diseñadas y la metodología empleada, se ha mostrado cómo determinadas propiedades de GeoGebra permiten al alumnado alcanzar niveles altos de perseverancia, autonomía, precisión-rigor, manejo del recurso y buen uso de las representaciones que este provee. También posibilita alcanzar niveles adecuados de flexibilidad de pensamiento, diseño de estrategias para resolver problemas y matematización en la mayoría de estudiantes. Actitudes como la creatividad y los procesos de pensamiento matemático de orden superior (razonamiento y prueba) se desarrollan en menor número de individuos y, además del software, necesitan la capacidad del docente para orquestar dinámicas de interacción apropiadas con estudiantes particulares en tiempo real. Esta labor se facilita en gran medida por la autonomía que otorga al alumnado el uso del SGD.

La ruta colectiva de interacción afecto-cognición identificada nos informa del desarrollo en el tiempo de las distintas actitudes y capacidades, así como de su estabilización en el alumnado. Básicamente, las actitudes hacia las matemáticas se desarrollan más rápidamente que las actitudes matemáticas, están vinculadas al uso del SGD y desaparecen tras su uso. Las actitudes matemáticas se desarrollan de modo más progresivo a partir de las anteriores y tienen más permanencia, aunque es complicado mantenerlas sin el apoyo de la herramienta. Todo el desarrollo actitudinal subyace tras las capacidades matemáticas, y tiene también una graduación en el tiempo: desde las manipulativas y visuales, estrechamente ligadas al manejo del software, hasta el pensamiento estratégico y los procesos de reflexión y argumentación, para los que cobra cada vez más relevancia la interacción social en el aula basada en su uso. Ello trae a colación la distinción entre las actitudes y capacidades que se desarrollan a través de la tecnología y aquellas que se manifiestan con la tecnología. Dicha distinción es más significativa en el caso de estudiantes con bajo perfil actitudinal y cognitivo. 
Por último, si bien la naturaleza contextualizada y en gran parte cualitativa de este estudio puede tomarse como una limitación, autores como Drijvers (2018) consideran indispensables los trabajos de este tipo y sus posibles replicaciones, por cuanto sirven de preliminar para identificar tendencias que informen el diseño de estudios a mayor escala. De esta forma, puede combinarse lo mejor de ambos planteamientos para atender a los retos educativos que se nos presentan.

\section{REFERENCIAS BIBLIOGRÁFICAS}

Baccaglini-Frank, A. (2019). Dragging, instrumented abduction and evidence, in processes of conjecture generation in a dynamic geometry environment. ZDM Mathematics Education. Online First, $1-13$. https://doi.org/10.1007/s11858-019-01046-8.

Bazán, J. (1997) Metodología estadística de construcción de pruebas. Una aplicación al estudio de actitudes hacia la matemática en la UNALM (tesis doctoral no publicada). UNALM, España.

Bray, A. y Tangney, B. (2017). Technology usage in mathematics education research - A systematic review of recent trends. Computers \& Education, 114, 255-273. https://doi.org/10.1016/j.compedu.2017.07.004.

Costa, J. (2011). Plataforma de matematización en un entorno GeoGebra dentro de un planteamiento didáctico «desde abajo hacia arriba». Enseñanza de las Ciencias, 29(1), 101-114. https://doi.org/10.5565/rev/ec/v29n1.527.

DeBellis, V. A. y Goldin, G. A. (2006). Affect and meta-affect in mathematical problem solving: A representational perspective. Educational Studies in Mathematics, 63(2), 131-147. https://doi.org/10.1007/s10649-006-9026-4.

Di Martino, P. y Zan, R. (2010). «Me and maths»: Towards a definition of attitude grounded on students' narratives. Journal of Mathematics Teacher Education, 13, 27-48. https://doi.org/10.1007/s10857-009-9134-z.

Drijvers, P. (2018). Empirical evidence for benefit? Reviewing quantitative research on the use of digital tools in mathematics education. En L. Ball, P. Drijvers, S. Ladel, H. S. Siller, M. Tabach, C. Vale (Eds), Uses of technology in primary and secondary mathematics education (pp. 161-175). Cham: Springer.

García, M. M. (2011). Evolución de actitudes y competencias matemáticas en estudiantes de secundaria al introducir GeoGebra en el aula (tesis doctoral no publicada). Universidad de Almería. http://funes. uniandes.edu.co/1768/2/Garcia2011Evolucion.pdf .

García, M. M. y Romero, I. M. (2020). Influencia de GeoGebra en las actitudes hacia las matemáticas de estudiantes de secundaria: diseño y validación de un cuestionario. En A. Codina y M. F. Moreno (Eds.), Investigaciones en Pensamiento Numérico y Algebraico: 2018 (pp. 83-100). Almería, España: Editorial de la Universidad de Almería.

Gómez Chacón, I. M. (2010). Actitudes de los estudiantes en el aprendizaje de la matemática con tecnología. Enseñanza de las Ciencias, 28(2), 227-244. https://doi.org/10.5565/rev/ec/v28n2.197.

Gómez-Chacón, I. M. (2011). Mathematics attitudes in computerized environments. A proposal using GeoGebra. En L. Bu y R. Schoen (Eds.), Model-centered learning: Pathways to mathematical understanding using GeoGebra (pp. 147-170). Rotterdam: Sense Publishers.

Gómez-Chacón, I. M. y Marbán, J. M. (2019). Afecto y conocimiento profesional docente en matemáticas. En E. Badillo, N. Climent, C. Fernández y M. T. González (Eds.), Investigación sobre el profesor de matemáticas: formación, práctica de aula, conocimiento y competencia profesional (pp. 397- 416). Salamanca: Ediciones Universidad Salamanca. 
Gómez-Chacón, I. M., Romero, I. M. y García, M. M. (2016). Zig-zagging in geometrical reasoning in technological collaborative environments: a Mathematical Working Space-framed study concerning cognition and affect. $Z D M, 48(6), 909-924$.

https://doi.org/10.1007/s11858-016-0755-2 .

Granberg, C. y Olsson, Y. (2015). ICT-supported problem solving and collaborative creative reasoning: Exploring linear functions using dynamic mathematics software. Journal of Mathematical Behavior, 37, 48-62.

https://doi.org/10.1016/j.jmathb.2014.11.001.

Gresalfi, M. S. (2009). Taking up opportunities to learn: constructing dispositions in mathematics classrooms. The Journal of the Learning Sciences, 18(3), 327-369.

https://doi.org/10.1080/10508400903013470.

Grootenboer, P. y Marshman, M. (2015). Mathematics, affect and learning. Singapur: Springer.

Hannula, M. S., Leder, G. C., Morselli, F., Vollstedt, M. y Zhang, Q. (Eds.) (2019). Affect and mathematics education. Cham: Springer.

Hannula, M., Pantziara, M. y Di Martino, P. (2018). Affect and mathematical thinking. Exploring developments, trends and future directions. En T. Dreyfus (Ed.), Developing research in mathematics education: twenty years of communication, cooperation, and collaboration in Europe (pp. 323-329). Abingdon, Oxon: Routledge.

Hoyles, C. y Lagrange, J. B. (2010). Mathematics education and technology: Rethinking the terrain. Nueva York: Springer.

Jaramillo, P. E. y Ruíz, M. (2010). El desarrollo de la autonomía: más allá del uso de las TIC para el trabajo independiente. Revista Colombiana de Educación, 58, 78-95.

https://doi.org/10.17227/01203916.637.

Lupiáñez, J. L. y Rico, L. (2008). Análisis didáctico y formación inicial de profesores: competencias y capacidades en el aprendizaje de los escolares. PNA, 3(1), 35-48.

Molina, M., Castro, E., Molina, J. L. y Castro, E. (2011). Un acercamiento a la investigación de diseńo a través de los experimentos de enseñanza. Enseñanza de las Ciencias, 29(1), 75-88. https://doi.org/10.5565/rev/ec/v29n1.435.

OECD (2010). PISA 2009 results: What students know and can do - Student performance in reading, mathematics and science (vol. I). París: OECD Publishing.

OECD (2017). Marco de evaluación y de análisis de PISA para el desarrollo: Lectura, matemáticas y ciencias (versión preliminar). París: OECD Publishing.

Rico, L. y Lupiáñez, J. L. (2008). Competencias matemáticas desde una perspectiva curricular. Madrid: Alianza Editorial.

Romero, I. M., García, M. M. y Codina, A. (2015). Developing mathematical competencies in secondary students by introducing dynamic geometry systems in the classroom. Eğitim ve Bilim, 40(177), 43-58. https://doi.org/10.15390/EB.2015.2640.

Roth, W. M. y Walshaw, M. (2019). Affect and emotions in mathematics education: Toward a holistic psychology of mathematics education. Educational Studies in Mathematics, 102(1), 111-125. https://doi.org/10.1007/s10649-019-09899-2.

Santos-Trigo, M. (2008). On the use of technology to represent and explore mathematical objects or problems dynamically. Mathematics and Computer Education, 42(2), 123-139.

Sinclair, N., Bussi, M. G. B., de Villiers, M., Jones, K., Kortenkamp, U., Leung, A. y Owens, K. (2016). Recent research on geometry education: An ICME-13 survey team report. ZDM, 48(5), 691-719.

https://doi.org/10.1007/s11858-016-0796-6. 
Sinclair, N. y Yurita, V. (2008). To be or to become: How dynamic geometry changes discourse. Research in Mathematics Education, 10(2), 135-150. https://doi.org/10.1080/14794800802233670.

Stolaki, A. y Economides, A. A. (2018). The creativity challenge game: An educational intervention for creativity enhancement with the integration of Information and Communication Technologies (ICTs). Computers \& Education, 123, 195-211. https://doi.org/10.1016/j.compedu.2018.05.009.

Takaci, D., Stankov, G. y Milanovic, I. (2015). Efficiency of learning environment using GeoGebra when calculus contents are learned in collaborative groups. Computers \& Education, 82, 421-431. https://doi.org/10.1016/j.compedu.2014.12.002

Wassie, Y. A. y Zergaw, G. A. (2018). Capabilities and contributions of the dynamic math software, GeoGebra-A review. North American GeoGebra Journal, 7(1), 68-86.

Yoganci, S. (2018). A study on the views of graduate students on the use of GeoGebra in mathematics teaching. European Journal of Education Studies, 4(8), 63-78.

https://doi.org/10.5281/zenodo.1272935

Zengìn, Y. (2017a). The potential of GeoGebra software for providing mathematical communication in the light of pre-service teachers' views. Necatibey Faculty of Education Electronic Journal of Science and Mathematics Education, 11(1), 101-127.

Zengìn, Y. (2017b). The effects of GeoGebra software on preservice mathematics teachers' attitudes and views toward proof and proving. International Journal of Mathematical Education in Science and Technology, 48(7), 1002-1022. https://doi.org/10.1080/0020739X.2017.1298855

Zetriuslita, Z., Nofriyandi, N. y Istikomah, E. (2020). The effect of Geogebra-assisted direct instruction on students' self-efficacy and self-regulation. Infinity, $9(1), 41-48$.

https://doi.org/10.22460/infinity.v9i1.p41-48 


\title{
Effects of working with GeoGebra in the classroom on the affect-cognition relationship
}

\author{
M. ${ }^{a}$ del Mar García López, Isabel M. ${ }^{a}$ Romero Albaladejo, Francisco Gil Cuadra \\ Departamento de Educación, Universidad de Almería,Almería, España. \\ mgl7II@ual.es, imromero@ual.es, fgil@ual.es
}

The rise of the dynamic approach to geometry requires well-founded empirical studies that inform its effective use in the classroom. To date, research mainly focuses on cognitive aspects, while the affective aspects and, furthermore, the affect-cognition relationship are paid less attention. This article responds to the demand for interventions that address both dimensions, as well as the relationship between them.

With respect to the attitudinal dimension, we distinguish between attitudes towards mathematics and mathematical attitudes. Within the first ones, we deal with motivation and self-confidence, which are considered the most influential in learning. Within the mathematical attitudes, which have a strong cognitive component and whose research is less developed, our work provides indicators for autonomy, creativity, flexibility of thought, perseverance and accuracy-rigor.

With regard to mathematical literacy, according to PISA framework (OECD, 2017), we consider 7 fundamental capabilities: communicating; mathematising; representing; reasoning and arguing; devising strategies for solving problems; using symbolic, formal and technical language and operations; and using mathematical tools.

Under the design research paradigm, a teaching experiment is conducted in which GeoGebra is introduced in two secondary school classes, with the purposes of (a) promoting the attitudinal development and the mathematical literacy of students, and (b) analysing the role of software in the evolution of the affective and cognitive dimensions, and the relationship between both of them.

Two didactic sequences were designed with the same methodological principles for the students to work in peers. The first one for pencil, paper and traditional instruments on polygons and the second one for GeoGebra on plane tessellations.

The data sources were questionnaires, teacher-researcher diaries, students' opinions, interviews, and observation grids for attitudes and mathematical capabilities (based on theory and endorsed by experts). Besides, the GeoGebra archives and audio recordings while working on the tasks were collected for a sample of students.

The quantitative analysis of the data shows a positive evolution in all the studied variables. The qualitative analysis (by means of the software Atlas.ti) informs about how this evolution took place, the properties of the software that supported it, and the relationship between the affective and cognitive constructs.

In our experiment, students' initial motivation and self-confidence with the use of GeoGebra helped them to develop motivation and confidence in doing mathematical work. These attitudes toward mathematics were linked to the work with GeoGebra and they disappeared after its use.

A good attitude towards mathematics, together with GeoGebra affordances, allowed the students in general to reach high levels in capabilities related to visualization, construction, and representations management. They also sustained the development of high degrees of perseverance, autonomy, and accuracy-rigor in the students. Mathematical attitudes emerged more progressively than attitudes towards mathematics and they were more permanent. Students continued to manifest them even after the experiment; nevertheless, a number of them could not keep them without the support of GeoGebra.

In due time, the majority of the students could also reach adequate levels in communication, flexibility of thought, devising strategies for solving problems, and mathematizing. On this basis, attitudes like creativity and higher order capabilities, such as logical reasoning and argument, were developed by a smaller number of individuals. In addition to the software affordances, the later development required the teacher to orchestrate proper interactions in the classroom. 